\title{
Identification of RAGE and OSM as New Prognosis Biomarkers of Severe Pneumonia
}

\author{
Jing Lei, Li Wang, Qian Li, Lin Gao, Jing Zhang, and Yan Tan (iD \\ Department of Respiratory and Critical Medicine, Nanjing First Hospital, Nanjing Medical University, Nanjing 210000, China \\ Correspondence should be addressed to Yan Tan; tanyan1970@126.com
}

Received 22 June 2021; Accepted 17 December 2021; Published 7 January 2022

Academic Editor: Jack Kastelik

Copyright (c) 2022 Jing Lei et al. This is an open access article distributed under the Creative Commons Attribution License, which permits unrestricted use, distribution, and reproduction in any medium, provided the original work is properly cited.

\begin{abstract}
Objective. To investigate efficiency of RAGE and OSM as new prognosis biomarkers of severe pneumonia. Methods. Eligible patients were classified into hypoxemia and nonhypoxemia groups. Meanwhile, the same cohort was divided into survival and nonsurvival groups after a post-hospital stay of 30 days. We analyzed risk factors for the hypoxia and death among these patients. Results. Compared with nonsurvival group, significant increase was noticed in PH, lymphocyte, albumin and platelet level in survival group, while significant decline was noticed in neutrophils, RBC, hemoglobin, hematocrit, creatinine, total bilirubin, CRP, PCT, OSM, RAGE and neutrophils/lymphocyte level. Oxygenation index level was related to APACHE II, LIS, SOFA, NUTRIC score, WBC, neutrophils, lymphocyte, RAGE, and albumin level $(p<0.05)$. LIS, SOFA, NUTRIC score, lac, lymphocyte, platelet, BUN, total bilirubin, PCT, and OSM levels were associated with mortality rate $(p<0.05)$. Conclusions. RAGE and OSM may serve as a new biomarker for poor prognosis in pneumonia patients.
\end{abstract}

\section{Introduction}

Pneumonia refers to a common disease that is usually associated with a poor treatment outcome. Patients with severe pneumonia usually show hypoxic respiratory failure, leading to a high morbidity and mortality. In the past decades, extensive efforts have been made on the diagnosis and treatment of pneumonia, in order to improve the outcome among these cases.

Increasing evidence indicates that laboratory biomarkers can be used for diagnosis and outcome prediction in infectious diseases and a variety of medical conditions including major cardiac events, cerebral hemorrhage, and cancer. Tamhane et al. proved that the neutrophil/lymphocyte ratio (NLR) was an independent predictor for mortality in patients who underwent percutaneous coronary intervention [1]. In addition, Lattanzi et al. [2] found that the NLR was associated with 30-day mortality and morbidity of patient with acute intracerebral hemorrhage, and improved the accuracy of outcome prediction. Howard et al. proved that NLR was associated with poorer survival outcomes in patients with solid tumor [3]. Moreover, patients with ischemic stroke who underwent endovascular treatment (EVT) showed higher systemic inflammatory response index (SIRI) at admission, and were at increased risk of poor outcome [4].

Patients with severe pneumonia usually show concomitant diseases such as cerebrovascular disease (CVD), diabetes mellitus (DM), coronary heart disease (CHD), chronic renal damage, and nasopharyngeal carcinoma. Indeed, the concomitant diseases closely associated with hypoxia have been acknowledged as risk factors for the progression of pneumonia. To date, there is still a lack of specific and sensitive markers for early identification of patients with increased risks of severe pneumonia, especially those complicated with hypoxic respiratory failure.

The receptor for advanced glycation end products (RAGE), a $35 \mathrm{kDa}$ protein from the immunoglobulin superfamily that propagates the inflammatory response via NF- $\kappa \mathrm{B}$ [5], is a marker of type I alveolar epithelial cell injury in rats and patients with acute respiratory distress syndrome (ARDS) [6]. In a previous study, plasma RAGE level showed decline in lung-protective ventilation in patients undergoing major abdominal surgery compared to those who received 
nonprotective ventilation [7]. Meanwhile, a high plasma RAGE was associated with an increased mortality in patients with acute lung injury [8]. Unfortunately, little is known about the roles of RAGE in the pathogenesis and prognosis of pneumonia. Oncostatin $M$ (OSM) receptors are a member of the IL-6 family, involving in endothelial damages that contribute to increased permeability to liquid and protein and consequent edema in interstitium space [9]. Neutrophilreleased OSM affected endothelial cellular function under both physiological and pathological conditions [10]. Despite its significance in pathogenesis of certain diseases, the feasibility of OSM as a biomarker for predicting the disease severity and prognosis is still not well defined. In this study, we investigated the feasibility of RAGE and OSM as new biomarkers for predicting the outcomes in patients with pneumonia.

\section{Materials and Methods}

2.1. Participants. This study was performed in the respiratory intensive care unit (RICU) of our hospital. All clinical data were retrospectively reviewed for pneumonia patients admitted to the RICU from January 2017 to October 2019. Patients included in our study presented at least one acute symptom (e.g. breathlessness, cough or fever). Besides, they showed pulmonary infiltration based on chest high resolution computed tomography. Patients with other lung complications such as tuberculosis, acute pulmonary embolism, congenital heart disease, and untreated aggressive carcinoma were excluded from this study.

2.2. Study Design. Patients were divided into two groups based on oxygenation index: group 1 with oxygenation index of $>250 \mathrm{mmHg}$ and group 2 with oxygenation index of $\leq 250 \mathrm{mmHg}$. After RICU admission, patient characteristics including disease course, medical history, smoking, drinking alcohol, physical and vital signs, hospital stay, prognosis, and medication histories were collected on days 1 and 7, respectively. Besides, the treatment options (e.g., oxygen usage and MV setting) were also collected. Furthermore, laboratory indices were measured, including blood cell count, coagulation analysis, CRP, PCT, OSM, RAGE, and T cell subtypes, as well as hepatic, renal, and cardiac tests.

We measured the lung injury score (LIS), NUTRIC score, gas exchange, as well as organ failure. Specifically, LIS was calculated based on oxygenation index, the area of pulmonary infiltration on chest X-ray, positive end expiratory pressure (PEEP), and pulmonary compliance. NUTRIC score was calculated based on age, APACHE II score, sequential organ failure assessment (SOFA), concomitant diseases, time from admission to RICU, and IL-6.

According to the prognosis, patients were separated into survival and nonsurvival groups. Patients with pneumonia that had different outcomes were compared in terms of clinical, laboratory and prognostic characteristics on day 1 and day 7 after admission. We then analyzed the related risk factors for the mortality.
2.3. Statistical Analysis. Statistical analysis was performed using SPSS 22.0 software. Continuous variables were presented as mean \pm standard deviation, and categorical variables were summarized as frequency and percentages. Independent-sample Student's $t$-test was utilized for intergroup comparison of continuous variables, while Chi-square test or Mann-Whitney test was used for intergroup comparison of categorical variables. Single factor Logistic regression analysis was conducted for the identification of risk factors. The area under receiver operating characteristic (ROC) curves indicated a strong predictive power for the biomarkers' area, which represented the largest area under the curve (AUC). Pearson product-moment correlation analysis was given to investigate the relationship between these markers and the survival or severity of pneumonia. $p<0.05$ was considered to be statistically significant.

\section{Results}

3.1. Patient Characteristics. Among the 828 cases admitted into the RICU of our hospital, 130 patients (15.70\%) were enrolled in our final analysis and were divided into group 1 with oxygenation index of $>250 \mathrm{mmHg}(n=76)$ and group 2 with oxygenation index of $\leq 250 \mathrm{mmHg}(n=54)$. There were no statistical differences in the baseline information of these patients in both groups. Among the patients, 16 were dead, and 112 survived.

3.2. Comparison of Gender and Concomitant Diseases, Age and Clinical Scores, and Clinical Scores. The proportion of cases with DM in the group with oxygenation index of $\leq 250 \mathrm{mmHg}$ was significantly higher compared with the group with oxygenation index of $>250 \mathrm{mmHg}$. In addition, the male was more likely to develop hypoxemia (Table 1). For the concomitant diseases, there were no statistical differences in the proportion of patients with CVD, CHD, chronic renal damage, and nasopharyngeal carcinoma between the two groups $(p>0.05$, Table 1$)$.

There were no statistical differences in age between two groups. APACHE II, LIS, SOFA and NUTRIC scores showed significant increase in the patients with an oxygenation index of $\leq 250 \mathrm{mmHg}$ compared to those with an oxygenation index of $>250 \mathrm{mmHg}(p<0.05$, Table 2$)$. Compared with patients with an oxygenation index of $>250 \mathrm{mmHg}$, there was significant increase in the white blood cell (WBC) count, neutrophils, neutrophils/lymphocyte ratio, lactic acid, creatinine, D-dimer, PCT, CRP and RAGE levels in patients with an oxygenation index of $\leq 250 \mathrm{mmHg} \quad(p<0.05$, Table 3). In contrast, the $\mathrm{PH}$, lymphocyte and albumin levels were significantly lower in the patients with an oxygenation index of $>250 \mathrm{mmHg}$ compared with the counterparts with an oxygenation index of $\leq 250 \mathrm{mmHg}(p<0.05)$. There were no significant differences in $\mathrm{PaCO}_{2}$, hematocrit, hemoglobin, platelet, erythrocyte, blood urea nitrogen (BUN), lactate dehydrogenase $(\mathrm{LDH})$, total bilirubin, prothrombin time (PT), activated partial thromboplastin time (APTT), fibrinogen (Fib) and OSM level between two groups. 
TABle 1: Gender and comorbidities.

\begin{tabular}{|c|c|c|c|c|}
\hline Variable & Oxygenation index $>250 \mathrm{mmHg}(n=54)$ & Oxygenation index $\leq 250 \mathrm{mmHg}(n=76)$ & $Z$ value & $p$ value \\
\hline Female & 21 & 16 & -2.212 & 0.027 \\
\hline Male & 33 & 60 & & \\
\hline Cerebrovascular disease & 11 & 26 & -1.717 & 0.086 \\
\hline Diabetes mellitus & 7 & 21 & -1.997 & 0.046 \\
\hline Coronary heart disease & 19 & 31 & -0.645 & 0.519 \\
\hline Chronic renal damage & 4 & 12 & -1.428 & 0.153 \\
\hline Treated nasopharyngeal carcinoma & 0 & 2 & -1.197 & 0.231 \\
\hline
\end{tabular}

TAble 2: Age, APACHE II, LIPS, SOFA, and NUTRIC scores.

\begin{tabular}{|c|c|c|c|c|}
\hline Variable & Oxygenation index $>250 \mathrm{mmHg}(n=54)$ & Oxygenation index $\leq 250 \mathrm{mmHg}(n=76)$ & $Z$ value & $p$ value \\
\hline Age & $69.63 \pm 16.17$ & $72.78 \pm 14.94$ & -1.144 & 0.255 \\
\hline APACHE II & $16.97 \pm 6.12(32)$ & $20.03 \pm 6.84(72)$ & -2.17 & 0.032 \\
\hline LIS & $3.13 \pm 2.24(32)$ & $7.04 \pm 2.78(74)$ & -7.032 & 0.0001 \\
\hline SOFA & $2.67 \pm 2.60(30)$ & $6.34 \pm 3.41(74)$ & -5.294 & 0.0001 \\
\hline NUTRIC score & $2.60 \pm 2.71(53)$ & $5.46 \pm 1.89(76)$ & -7.069 & 0.0001 \\
\hline
\end{tabular}

TABLE 3: Laboratory examination results at RICU admission.

\begin{tabular}{|c|c|c|c|c|}
\hline Variable & Oxygenation index $>250 \mathrm{mmHg}(n=54)$ & Oxygenation index $\leq 250 \mathrm{mmHg}(n=76)$ & $Z$ value & $p$ value \\
\hline \multicolumn{5}{|l|}{ Immunophenotyping } \\
\hline $\mathrm{CD} 4$ & $462.79 \pm 171.08(28)$ & $421.08 \pm 123.61(65)$ & 1.324 & 0.189 \\
\hline CD8 & $259.79 \pm 98.31(28)$ & $259.49 \pm 107.88(65)$ & 0.012 & 0.990 \\
\hline CD3 & $809.93 \pm 239.67(28)$ & $764.83 \pm 201.67(64)$ & 0.931 & 0.354 \\
\hline \multicolumn{5}{|l|}{ Arterial blood gas } \\
\hline $\mathrm{pH}$ & $7.44 \pm 0.45(50)$ & $7.41 \pm 0.09(76)$ & 1.850 & 0.067 \\
\hline $\mathrm{PaCO} 2$ (mmHg) & $38.56 \pm 7.05(50)$ & $41.66 \pm 17.25(76)$ & -1.206 & 0.230 \\
\hline $\mathrm{Lac}$ & $1.47 \pm 0.80(50)$ & $1.88 \pm 1.39(76)$ & -1.880 & 0.063 \\
\hline \multicolumn{5}{|l|}{ Blood cell analysis } \\
\hline WBC $(109 / \mathrm{L})$ & $8.09 \pm 4.16(53)$ & $10.71 \pm 6.10(76)$ & -2.717 & 0.008 \\
\hline Neutrophils (109/L) & $6.09 \pm 3.83(53)$ & $9.31 \pm 5.71(76)$ & -3.581 & $\leq 0.001$ \\
\hline Lymphocyte $\left(10^{9} / \mathrm{L}\right)$ & $1.28 \pm 0.80(53)$ & $0.86 \pm 0.53(76)$ & 3.555 & 0.001 \\
\hline Hematocrit (\%) & $0.37 \pm 0.07$ & $0.37 \pm 0.08(76)$ & -0.277 & 0.782 \\
\hline Hemoglobin $(\mathrm{g} / \mathrm{L})$ & $122.60 \pm 21.85(53)$ & $121.04 \pm 27.05(76)$ & 0.349 & 0.728 \\
\hline Platelet $\left(10^{9} / \mathrm{L}\right)$ & $199.74 \pm 74.08(53)$ & $199.16 \pm 105.09(76)$ & 0.034 & 0.973 \\
\hline Erythrocyte $\left(10^{12} / \mathrm{L}\right)$ & $4.07 \pm 0.78$ & $4.10 \pm 0.94(76)$ & -0.142 & 0.888 \\
\hline Neutrophils/Lymphocyte (\%) & $9.32 \pm 16.40(51)$ & $15.13 \pm 15.98(76)$ & -1.989 & 0.049 \\
\hline \multicolumn{5}{|l|}{ Biochemical analysis } \\
\hline Albumin $(\mathrm{g} / \mathrm{L})$ & $35.80 \pm 7.70(51)$ & $30.38 \pm 4.53(75)$ & 4.962 & $\leq 0.001$ \\
\hline BUN (mmol/L) & $6.79 \pm 5.08(52)$ & $19.66 \pm 59.82(76)$ & -1.545 & 0.125 \\
\hline Creatinine $(\mu \mathrm{mol} / \mathrm{L})$ & $87.43 \pm 96.15(52)$ & $123.60 \pm 102.80(76)$ & -2.007 & 0.047 \\
\hline LDH (IU/L) & $375.90 \pm 178.88(51)$ & $542.54 \pm 716.54(76)$ & -1.625 & 0.107 \\
\hline Total bilirubin $(\mu \mathrm{mol} / \mathrm{L})$ & $12.17 \pm 5.63$ & $12.45 \pm 10.06(75)$ & -0.184 & 0.854 \\
\hline \multicolumn{5}{|l|}{ Coagulation analysis } \\
\hline PT (s) & $12.61 \pm 4.10(52)$ & $12.85 \pm 2.48(76)$ & -0.405 & 0.686 \\
\hline APTT(s) & $29.97 \pm 5.91(52)$ & $30.67 \pm 7.01(76)$ & -0.587 & 0.558 \\
\hline $\mathrm{FiB}(\mathrm{g} / \mathrm{L})$ & $4.54 \pm 1.60(52)$ & $5.35 \pm 4.69(76)$ & -1.194 & 0.235 \\
\hline D-dimer & $1.31 \pm 1.91(52)$ & $5.66 \pm 8.68(76)$ & -3.549 & 0.001 \\
\hline \multicolumn{5}{|l|}{ Inflammatory biomarkers } \\
\hline Procalcitonin $(\mathrm{ng} / \mathrm{mL})$ & $0.61 \pm 1.60$ & $6.98 \pm 20.91(71)$ & -2.105 & 0.037 \\
\hline CRP (ng/L) & $59.83 \pm 64.33(51)$ & $113.59 \pm 96.67(76)$ & -3.487 & 0.001 \\
\hline OSM (pg/mg) & $54.85 \pm 58.64(53)$ & $80.32 \pm 110.43(74)$ & -1.530 & 0.128 \\
\hline RAGE (pg/ml) & $632.62 \pm 469.73(53)$ & $1034.26 \pm 1068.24$ & -2.563 & 0.012 \\
\hline
\end{tabular}

3.3. Comparison of Parameters between the Survival and Nonsurvival Groups. Table 4 showed the comparison of gender and concomitant diseases between the survival and nonsurvival groups. There were no statistical differences in the gender, CVD, DM, CHD, chronic renal damage, and nasopharyngeal carcinoma between two groups $(p>0.05)$. For the comparison of age and clinical scores between the two groups, there was no statistical difference in age and 
TABLE 4: Gender and comorbidities between survival and nonsurvival patients.

\begin{tabular}{|c|c|c|c|c|}
\hline Variable & Non-survival group $(n=16)$ & Survival group $(n=114)$ & $Z$ value & $p$ value \\
\hline Female & 3 & 34 & -0.916 & 0.360 \\
\hline Male & 16 & 80 & & \\
\hline Cerebrovascular disease & 1 & 36 & -2.095 & 0.086 \\
\hline Diabetes mellitus & 5 & 23 & -1.005 & 0.315 \\
\hline Coronary heart disease & 9 & 41 & -1.556 & 0.120 \\
\hline Chronic renal damage & 3 & 13 & -0.834 & 0.404 \\
\hline Treated nasopharyngeal carcinoma & 0 & 2 & -0.532 & 0.595 \\
\hline
\end{tabular}

APACHE II score between two groups $(p>0.05)$. In contrast, the LIS, SOFA, and NUTRIC scores were significantly higher in the nonsurvival group compared to those of the survival group $(p<0.05$, Table 5$)$. Compared to the survival group, significant increase was seen in the levels of lactic acid $(p=0.008)$, BUN $(p=0.002)$, total bilirubin $(p=0.009)$, $\operatorname{PCT}(p=0.012)$, and OSM $(p=0.001)$ in the nonsurvival group (Table 6).

3.4. Comparison of Laboratory Findings in Survival or Nonsurvival Groups before and after Treatment. Tables 7 and 8 summarize the changes of laboratory findings before and after treatment in the survival and non-survival groups, respectively. Compared with the baseline levels, the $\mathrm{PH}$, lymphocyte, albumin, and platelet levels showed significant increase after treatment in the survival group $(p<0.05)$, while the number of neutrophils, red blood cells (RBCs), hemoglobin, hematocrit, creatinine, total bilirubin, CRP, PCT, OSM, RAGE, and neutrophils/lymphocyte ratio showed significant decrease compared with the baseline levels $(p<0.05)$.

It is important to point out that even though CRP and $\mathrm{PaCO}_{2}$ raised similarly to the survival group, the $\mathrm{PH}$ went lower in the nonsurvival group after treatment $(p<0.05)$. Meanwhile, Lac, WBC, neutrophils, lymphocyte, RBC, hemoglobin, hematocrit, platelet, albumin, creatinine, BUN, total bilirubin, PT, APTT, FiB, PCT, OSM, RAGE, and neutrophils/lymphocyte ratio showed no significant changes after treatment in the nonsurvival group.

3.5. Factors Correlated with Oxygenation Index and Survival. In this section, we determined the relationship between the oxygenation index and a serial of variables including scores of APACHE II, LIS, SOFA, NUTRIC, PH, lac acid, WBC, neutrophils, lymphocyte, neutrophils/lymphocyte, creatinine, RAGE, and albumin, respectively. Our data indicated that the oxygenation index was correlated with APACHE II, LIS, SOFA, NUTRIC scores, WBC, neutrophils, lymphocyte, RAGE, and albumin levels, respectively $(p<0.05)$. In addition, LIS, SOFA, NUTRIC scores, lactic acid, lymphocyte, platelet, BUN, total bilirubin, PCT, and OSM levels were correlated with the mortality $(p<0.05)$.

3.6. Correlation between Oxygenation Index and RAGE. To investigate the relationship between oxygenation index and RAGE, we calculated the Pearson correlation coefficient.
Figure 1 shows that oxygenation index was negatively correlated with RAGE $(r=-0.228, p=0.001)$.

3.7. Correlation between Pneumonia Mortality with OSM. OSM level was correlated with the pneumonia mortality before treatment $(r=-0.228, p=0.001$ Figure 2). At the baseline level, BUN, bilirubin, and platelet levels were correlated with survival. Nevertheless, CRP and PCT were correlated with survival after treatment.

For the cutoff value of BUN prior to treatment, AUC of BUN was 0.738 (95\% CI: 0.607-0.869, $p=0.002$ ) with a cutoff point of $9.22 \mathrm{mmol} / \mathrm{L}$. This yielded a sensitivity and specificity of $81.3 \%$ and $64.3 \%$, respectively (Figure 3 ).

ROC curves resulted in an AUC of 0.69 for serum total bilirubin (95\% CI: $0.557-0.824, p=0.014$ ) and 0.288 for platelet (95\% CI: $0.128-0.449, p=0.006$ ), respectively. The cutoff value for serum total bilirubin was $8.55 \mu \mathrm{mol} / \mathrm{L}$ with a sensitivity of $93.8 \%$ and specificity of $40.9 \%$. For the platelet, the cutoff value was $28 \times 10^{9} / \mathrm{L}$ with a sensitivity and specificity of $100 \%$ and $0 \%$, respectively (Figure 4 ).

3.8. Cutoff Value ofCRP and PCTafter Treatment. To identify the risk factors for mortality, we calculated the AUC of CRP and PCT after treatment, which yielded an AUC of 0.868 (95\% CI: $0.712-1.000, p=0.003)$ and 0.855 (95\% CI: $0.733-0.977, p=0.004)$, respectively. The cutoff values were $70.15 \mathrm{mg} / \mathrm{L}$ and $0.24 \mathrm{ng} / \mathrm{ml}$. The sensitivity and specificity for CRP was $83.3 \%$ and $87.9 \%$, while that for PCT was $100 \%$ and $72.4 \%$, respectively (Figure 5).

\section{Discussion}

A large number of patients with severe pneumonia present hypoxic respiratory failure, which results in a high morbidity and mortality. In the past decades, extensive efforts have been made on the diagnosis and treatment of pneumonia with an aim to improve the outcome. In this study, we examined the feasibility of RAGE and OSM in predicting the outcome of pneumonia. In this retrospective study, our data showed that pneumonia with a lower oxygenation index was associated with gender, DM, APACHE II score, LIS, SOFA, NUTRIC score, PH value, lactic acid, WBC, neutrophils, lymphocyte, neutrophils/lymphocyte count, creatinine, D-dimer, PCT, CRP, RAGE, and albumin levels. Among these factors, the APACHE II score, LIS, SOFA, NUTRIC scores, WBC, neutrophils, lymphocyte count, 
TABle 5: Age, APACHE II, LIPS, SOFA and NUTRIC scores between survival and nonsurvival patients.

\begin{tabular}{|c|c|c|c|c|}
\hline Variable & Non-survival group $(n=16)$ & Survival group $(n=114)$ & $Z$ value & $p$ value \\
\hline Age & $73.44 \pm 12.36$ & $71.19 \pm 15.89$ & 0.542 & 0.589 \\
\hline APACHE II & $20.93 \pm 6.02(15)$ & $18.77 \pm 6.84(89)$ & 1.148 & 0.254 \\
\hline LIS & $7.56 \pm 3.93(16)$ & $5.56 \pm 2.95(90)$ & 2.376 & 0.019 \\
\hline SOFA & $7.69 \pm 5.04(16)$ & $4.84 \pm 3.11(113)$ & 3.022 & 0.003 \\
\hline NUTRIC score & $7.69 \pm 5.04(16)$ & $4.09 \pm 2.68(113)$ & 2.292 & 0.024 \\
\hline
\end{tabular}

TABLE 6: Laboratory examination results between survival and nonsurvival patients before treatment.

\begin{tabular}{|c|c|c|c|c|}
\hline Variable & Non-survival group $(n=16)$ & Survival group $(n=114)$ & $Z$ value & $p$ value \\
\hline $\begin{array}{l}\text { Immunophenotyping } \\
\text { CD4 } \\
\text { CD8 } \\
\text { CD3 }\end{array}$ & $\begin{array}{l}381.33 \pm 35.13(12) \\
221.50 \pm 26.78(12) \\
681.33 \pm 77.79(12)\end{array}$ & $\begin{array}{l}441.38 \pm 147.94(81) \\
265.22 \pm 110.56(81) \\
793.14 \pm 223.77(80)\end{array}$ & $\begin{array}{l}-1.394 \\
-1.358 \\
-1.708\end{array}$ & $\begin{array}{l}0.167 \\
0.178 \\
0.091\end{array}$ \\
\hline $\begin{array}{l}\text { Arterial blood gas } \\
\mathrm{pH} \\
\mathrm{PaCO} 2(\mathrm{mmHg}) \\
\mathrm{Lac}\end{array}$ & $\begin{array}{l}7.44 \pm 0.08(16) \\
39.59 \pm 8.41(16) \\
2.46 \pm 2.13(16) \\
\end{array}$ & $\begin{array}{c}7.42 \pm 0.08(110) \\
40.55 \pm 14.83(110) \\
1.61 \pm 0.98(110) \\
\end{array}$ & $\begin{array}{c}1.074 \\
-0.252 \\
2.676\end{array}$ & $\begin{array}{l}0.285 \\
0.801 \\
0.008 \\
\end{array}$ \\
\hline $\begin{array}{l}\text { Blood cell analysis } \\
\text { WBC }\left(10^{9} / \mathrm{L}\right) \\
\text { Neutrophils }\left(10^{9} / \mathrm{L}\right) \\
\text { Lymphocyte }\left(10^{9} / \mathrm{L}\right) \\
\text { Hematocrit }(\%) \\
\text { Hemoglobin }(\mathrm{g} / \mathrm{L}) \\
\text { Platelet }(109 / \mathrm{L}) \\
\text { Erythrocyte }\left(10^{12} / \mathrm{L}\right) \\
\text { Neutrophils/Lymphocyte (\%) }\end{array}$ & $\begin{array}{c}8.30 \pm 4.63(16) \\
7.26 \pm 4.55(16) \\
0.73 \pm 0.59(16) \\
0.35 \pm 0.08(16) \\
115.56 \pm 25.18(16) \\
139.88 \pm 81.63(16) \\
3.74 \pm 0.72(16) \\
13.94 \pm 12.15(16)\end{array}$ & $\begin{array}{c}9.83 \pm 5.63(113) \\
8.09 \pm 5.36(113) \\
1.078 \pm 0.69(113) \\
0.37 \pm 0.08(113) \\
122.55 \pm 24.93(113) \\
207.82 \pm 92.05(113) \\
4.13 \pm 0.89(113) \\
12.63 \pm 16.89(111)\end{array}$ & $\begin{array}{c}-1.034 \\
-0.587 \\
-1.941 \\
-0.852 \\
-1.048 \\
-2.799 \\
-1.691 \\
0.298\end{array}$ & $\begin{array}{l}0.303 \\
0.558 \\
0.054 \\
0.396 \\
0.297 \\
0.006 \\
0.093 \\
0.766\end{array}$ \\
\hline $\begin{array}{l}\text { Biochemical analysis } \\
\text { Albumin }(\mathrm{g} / \mathrm{L}) \\
\text { BUN }(\mathrm{mmol} / \mathrm{L}) \\
\text { Creatinine }(\mu \mathrm{mol} / \mathrm{L}) \\
\mathrm{LDH}(\mathrm{IU} / \mathrm{L}) \\
\text { Total bilirubin }(\mu \mathrm{mol} / \mathrm{L})\end{array}$ & $\begin{array}{c}33.59 \pm 11.41(16) \\
48.27 \pm 128.46(16) \\
113.06 \pm 81.79(16) \\
710.81 \pm 948.51(16) \\
17.49 \pm 12.15(16)\end{array}$ & $\begin{array}{c}32.43 \pm 5.59(110) \\
9.60 \pm 7.56(112) \\
108.31 \pm 104.13(112) \\
441.72 \pm 490.33(111) \\
11.59 \pm 7.65(110)\end{array}$ & $\begin{array}{l}0.664 \\
3.223 \\
0.175 \\
1.780 \\
2.651 \\
\end{array}$ & $\begin{array}{l}0.508 \\
0.002 \\
0.862 \\
0.071 \\
0.009\end{array}$ \\
\hline $\begin{array}{l}\text { Coagulation analysis } \\
\text { PT }(\mathrm{s}) \\
\mathrm{APTT}(\mathrm{s}) \\
\mathrm{FiB}(\mathrm{g} / \mathrm{L}) \\
\text { D-dimer }\end{array}$ & $\begin{array}{c}13.23 \pm 3.25(16) \\
31.43 \pm 9.16(16) \\
3.85 \pm 2.23(16) \\
6.12 \pm 8.74(16)\end{array}$ & $\begin{array}{c}12.68 \pm 3.23(112) \\
30.24 \pm 6.15(112) \\
5.19 \pm 3.91(112) \\
3.58 \pm 6.83(112)\end{array}$ & $\begin{array}{c}0.626 \\
0.675 \\
-1.328 \\
1.342\end{array}$ & $\begin{array}{l}0.533 \\
0.501 \\
0.187 \\
0.182\end{array}$ \\
\hline $\begin{array}{l}\text { Inflammatory biomarkers } \\
\text { Procalcitonin }(\mathrm{ng} / \mathrm{mL}) \\
\text { CRP }(\mathrm{ng} / \mathrm{L}) \\
\text { OSM }(\mathrm{pg} / \mathrm{mg}) \\
\text { RAGE }(\mathrm{pg} / \mathrm{ml})\end{array}$ & $\begin{array}{c}14.68 \pm 36.15(14) \\
88.24 \pm 107.42(16) \\
142.63 \pm 196.72(15) \\
1099.89 \pm 810.56(15)\end{array}$ & $\begin{array}{c}3.04 \pm 11.28(105) \\
92.54 \pm 86.45(111) \\
59.92 \pm 64.15(112) \\
835.41 \pm 898.62(112)\end{array}$ & $\begin{array}{c}2.545 \\
-0.180 \\
3.366 \\
1.082\end{array}$ & $\begin{array}{l}0.012 \\
0.857 \\
0.001 \\
0.281\end{array}$ \\
\hline
\end{tabular}

RAGE and albumin levels were independent risk factors for severe pneumonia.

RAGE is constitutively highly expressed in type 1 and type 2 alveolar epithelial cells and vascular smooth muscle cells in lung [11]. It has been well accepted that RAGE is defined as a specific marker of ARDS [7]. For instance, RAGE can be targeted as new therapeutic strategies for the management of ARDS patients. [12] Besides, RAGE was closely associated with the pathogenesis of hypoxic pneumonia [13]. In our study, the increased serum RAGE level was an independent risk factor for hypoxemia in pneumonia patients. Our data suggested that RAGE might serve as a new biomarker for predicting hypoxemia of pneumonia even before the onset of ARDS.
Patients with severe pneumonia show a high morbidity and mortality. On this basis, screening of patients with high risks of severe pneumonia contributes to the early diagnosis and/or intervention, as well as delay in disease progression and even death. Some studies reported that lactate and lymphocyte could predict mortality in injured patients after resuscitation [14, 15]. Our data showed that LIS, SOFA, NUTRIC scores, lactic acid, lymphocyte, platelet, erythrocyte, BUN, LDH, total bilirubin, PCT, and OSM levels were significantly different between two groups. Meanwhile, LIS, SOFA scores, lactate, lymphocyte, platelet, BUN, total bilirubin, PCT, and OSM levels were proved to be independent predictive factors for a high mortality before treatment. 
TABLE 7: Laboratory examination results before and after one-week treatment in the survival group.

\begin{tabular}{lccc}
\hline Variable & Survival & $T$ value & $p$ value \\
\hline Lac & $-0.77 \pm 9.64$ & -0.692 & 0.491 \\
$\mathrm{PH}$ & $-0.03 \pm 0.08$ & -3.184 & 0.002 \\
$\mathrm{PaCO}_{2}$ & $-1.81 \pm 13.27$ & -1.173 & 0.245 \\
WBC & $1.11 \pm 6.24$ & 1.536 & 0.129 \\
Neutrophils & $1.52 \pm 6.00$ & 2.182 & 0.032 \\
Lymphocyte & $-0.22 \pm 0.82$ & -2.340 & 0.022 \\
RBC & $0.41 \pm 0.56$ & 6.271 & $\leq 0.001$ \\
Hemoglobin & $17.79 \pm 17.26$ & 6.602 & $\leq 0.001$ \\
Hematocrit & $0.04 \pm 0.05$ & 5.761 & $\leq 0.001$ \\
Platelet & $-66.54 \pm 100.87$ & -4.224 & $\leq 0.001$ \\
Albumin & $-3.70 \pm 5.45$ & -4.344 & $\leq 0.001$ \\
Creatinine & $23.79 \pm 53.28$ & 2.859 & 0.007 \\
BUN & $-0.26 \pm 6.15$ & -0.274 & 0.785 \\
Total bilirubin & $3.39 \pm 9.91$ & 2.187 & 0.035 \\
PT & $0.30 \pm 3.57$ & 0.533 & 0.597 \\
APTT & $0.47 \pm 6.89$ & 0.440 & 0.663 \\
FiB & $1.62 \pm 6.16$ & 1.685 & 0.100 \\
CRP & $92.20 \pm 77.36$ & 7.632 & $\leq 0.001$ \\
PCT & $5.02 \pm 12.87$ & 2.501 & 0.017 \\
OSM & $43.64 \pm 68.11$ & 5.086 & $\leq 0.001$ \\
RAGE & $379.09 \pm 891.45$ & 3.375 & 0.001 \\
Neutrophils/Lymphocyte & $6.71 \pm 14.86$ & 3.587 & 0.001 \\
\hline
\end{tabular}

TABLE 8: Laboratory examination results before and after one-week treatment in the nonsurvival group.

\begin{tabular}{lccc}
\hline Variable & Survival & $T$ value & $p$ value \\
\hline Lac & $-2.41 \pm 4.31$ & -1.853 & 0.094 \\
$\mathrm{PH}$ & $-0.150 \pm 0.13$ & 3.878 & 0.003 \\
$\mathrm{PaCO}_{2}$ & $-16.20 \pm 19.20$ & -2.799 & 0.019 \\
WBC & $-1.85 \pm 4.36$ & -1.409 & 0.189 \\
Neutrophils & $-2.21 \pm 4.11$ & -1.409 & 0.189 \\
Lymphocyte & $0.38 \pm 0.79$ & 1.579 & 0.145 \\
RBC & $0.40 \pm 0.66$ & 2.020 & 0.071 \\
Hemoglobin & $18.40 \pm 22.14$ & 1.858 & 0.137 \\
Hematocrit & $0.05 \pm 0.06$ & 1.783 & 0.149 \\
Platelet & $-36.20 \pm 86.04$ & -0.941 & 0.400 \\
Albumin & $3.92 \pm 5.87$ & 1.492 & 0.210 \\
Creatinine & $-68.80 \pm 94.59$ & -1.626 & 0.179 \\
BUN & $92.38 \pm 234.38$ & 0.867 & 0.435 \\
Total bilirubin & $-14.22 \pm 26.91$ & -1.182 & 0.303 \\
PT & $-2.08 \pm 1.97$ & -2.359 & 0.078 \\
APTT & $-4.52 \pm 5.79$ & -1.746 & 0.156 \\
FiB & $-2.42 \pm 2.66$ & -2.032 & 0.112 \\
CRP & $-73.86 \pm 48.48$ & -3.406 & 0.027 \\
PCT & $-9.74 \pm 21.28$ & -1.023 & 0.364 \\
OSM & $-14.73 \pm 50.73$ & -0.711 & 0.509 \\
RAGE & $163.81 \pm 1544.84$ & 0.260 & 0.805 \\
Neutrophils/Lymphocyte & $-10.36 \pm 19.58$ & -1.296 & 0.252 \\
\hline
\end{tabular}

BUN was reported to be independently associated with mortality in critically ill patients [16]. Our data showed that the cutoff value of BUN was $9.22 \mathrm{mmol} / \mathrm{L}$, which showed a sensitivity and specificity of $81.3 \%$ and $64.3 \%$ for the prediction of mortality. As previously described, elevation of serum bilirubin was associated with ARDS development and mortality in sepsis [17]. The cutoff value of serum bilirubin was $8.55 \mu \mathrm{mol} / \mathrm{L}$ in our study, while the sensitivity and specificity of possibility for

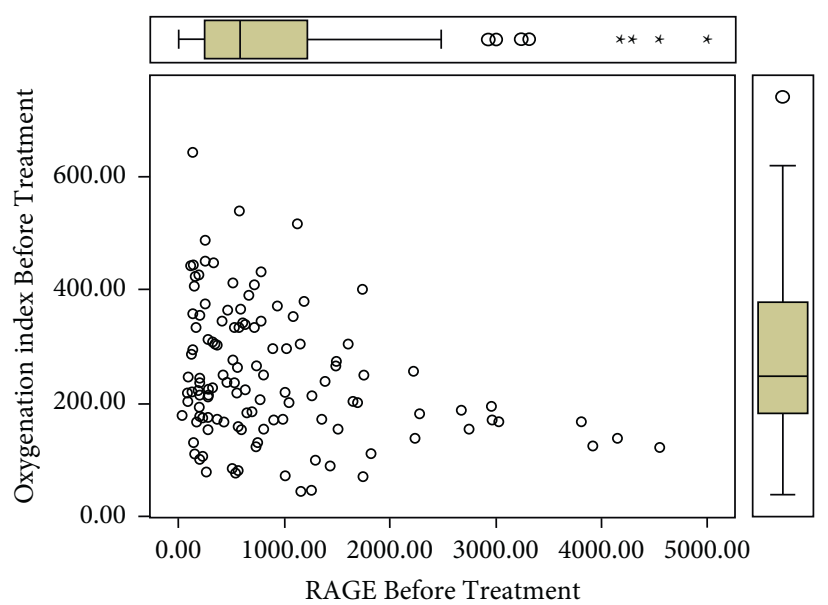

FIGURE 1: In order to examine the relationship between oxygenation index and the RAGE, we calculated the Pearson correlation coefficient, with $-0.228, p=0.001$ indicating a meaningful negative correlation between RAGE and oxygenation index.

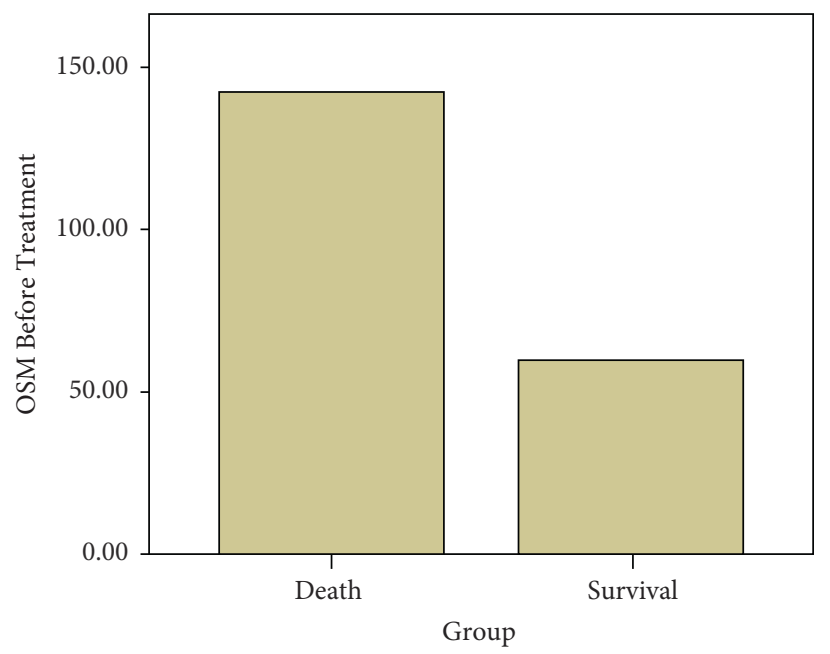

FIGURE 2: OSM level before treatment was associated with the pneumonia mortality. The Pearson correlation coefficient was -0.228 , and $p=0.001$.

death was $93.8 \%$ and $40.9 \%$, respectively. Moreover, posttreatment PCT and CRP, rather than pre-treatment PCT and CRP, were independent risk factors for 30-day mortality. Single factor logistic regression analysis revealed a cutoff value of $0.24 \mathrm{ng} / \mathrm{ml}$ for PCT, with a sensitivity of $100 \%$ and a specificity of $72.4 \%$, respectively. In addition, the cutoff value for CRP was $70.15 \mathrm{mg} / \mathrm{L}$, with a sensitivity of $83.3 \%$ and a specificity of $87.9 \%$. These indicated that post-treatment PCT and CRP were risk factors for death. To date, there are still disputes on the roles of PCT in predicting the outcome of pneumonia. For example, some studies indicated that PCT was not an independent predictor of 30-day mortality in elderly and younger patients [18]. In contrast, some studies indicated that elevated PCT had moderate accuracy to identify poor outcome in septic patients [19]. However, we were among the very few groups that studied the relationship of PCT with disease morbidity after treatment. 


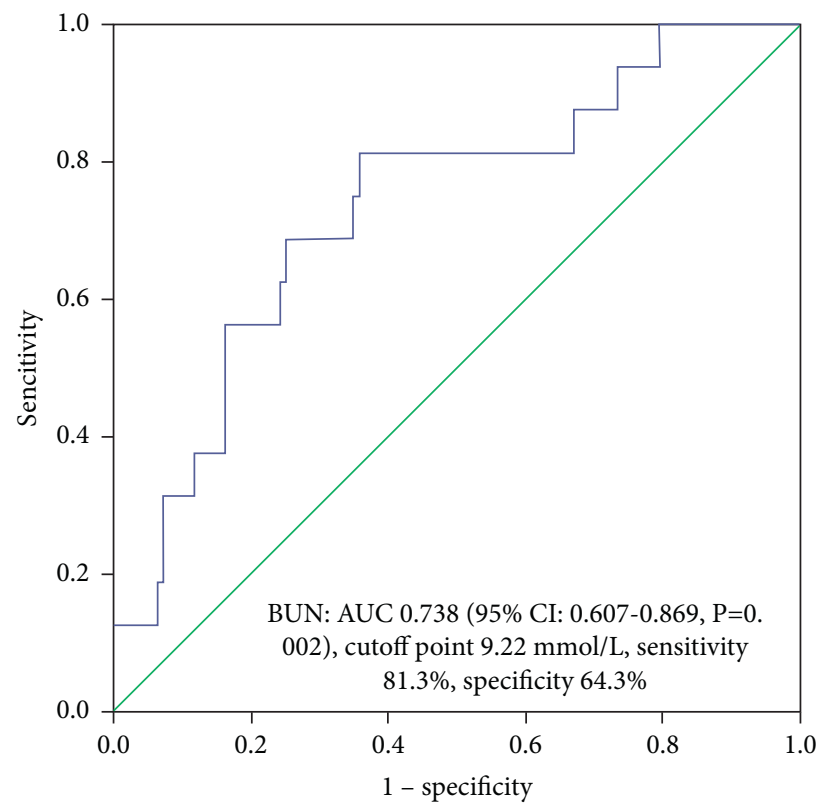

Figure 3: The AUC of BUN before treatment was calculated to be 0.738 ( $95 \% \mathrm{Cl}: 0.607-0.869, p=0.002)$ with a cutoff point of $9.22 \mathrm{mmol} / \mathrm{L}$ yielding a sensitivity and specificity of $81.3 \%$ and $64.3 \%$, respectively.

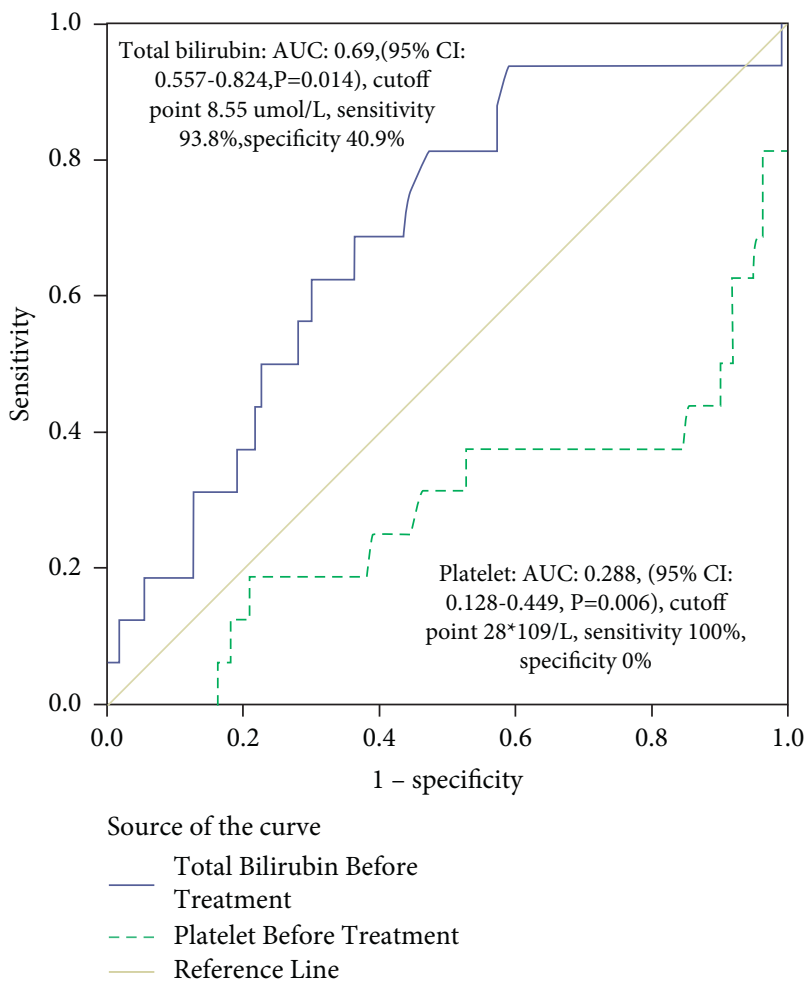

FIGURE 4: ROC curves of serum total bilirubin and platelet resulted in an AUC of 0.69 (95\% CI: 0.557-0.824, $p=0.014)$ and $0.288(95 \%$ CI: $0.128-0.449, p=0.006)$, respectively, using a cutoff value of $8.55 \mathrm{umol} / \mathrm{L}$ for serum total bilirubin with a sensitivity of $93.8 \%$ and specificity of $40.9 \%$, and a cutoff value of $28\left({ }^{*} 10^{9} / \mathrm{L}\right)$ for platelet with a sensitivity and specificity of $100 \%$ and $0 \%$.

In this study, we did not test the level of interleukin directly; however, the previous studies have shown that interleukin is closely related to the severity of pneumonia.

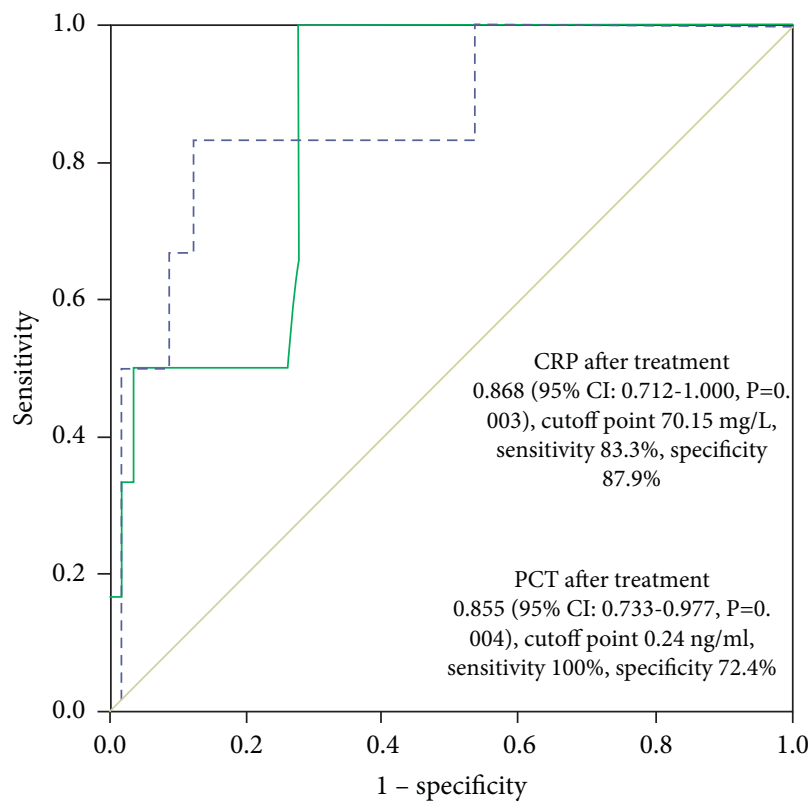

Source of the curve

- - - CRP After Treatment

— PCT After Treatment

_ Reference Line

FIGURE 5: In order to identify the risk for death, we calculated the AUC of CRP and PCT after treatment which were 0.868 (95\% Cl: $0.712-1.000, p=0.003)$ and 0.855 (95\% Cl: 0.733-0.977, $p=0.004)$ with cutoff values being $70.15 \mathrm{mg} / \mathrm{L}$ and $0.24 \mathrm{ng} / \mathrm{ml}$, respectively. The sensitivity/specificity was $83.3 \% / 87.9 \%$ and $100 \% / 72.4 \%$, respectively.

For example, Han et al. showed that patients with COVID19 pneumonia have higher serum level of cytokines (TNF- $\alpha$, IFN- $\gamma$, IL-2, IL-4, IL-6 and IL-10) and CRP than control individuals. Serum IL-6 and IL-10 levels are significantly 
higher in the critical group than in the moderate and severe groups [20]. Also, Diane et al. found that high serum IL-6, IL-8, and TNF- $\alpha$ levels were strong and independent predictors of patient survival with COVID-19 pneumonia at the time of hospitalization [21]. As a member of the IL-6 family cytokines, OSM is closely involved in response to bacterial stimuli. However, little is known about its role in pneumonia. In this study, increased serum OSM at admission was associated with elevation of 30-day mortality. In a previous study, OSM may function as an important signal to epithelial cells for chemokines induction mediating neutrophil recruitment, which finally triggered the pathogenesis of severe inflammation [22]. Besides, it was also associated with increased vessel permeability as it could lead to endothelial barrier damages. Furthermore, OSM can also contribute to the activation of fibroblasts as well as multiple organ dysfunction during severe infection [23]. Consistently, our study showed that OSM was associated with prognosis of pneumonia. In future, more studies are needed to further confirm the predictive value of OSM in clinical practice.

It has been found that RAGE is related to OSM. Arunachalam et al. proved that there were enhanced plasma levels of inflammatory mediators, including EN-RAGE, TNFSF 14, and oncostatin-M (OSM) in severe COVID-19 infected patients, which was correlated with disease severity and increased bacterial products [24]. Moreover, knockout of receptor for advanced RAGE significantly attenuated cigarette smoke-induced airway inflammation in mice, and functional enrichment analyses showed the 14 functional methylated genes were enriched in immune-inflammatory responses, especially interleukin IL-6 and IL-17 pathways. This study suggested that RAGE mediated functional DNA methylated modification in a cluster of 14 targeted genes, particularly hypomethylation in promoter of OSM [25]. Therefore, we will focus on the correlation between OSM and RAGE in severe pneumonia.

There are really some limitations in our study. First, this was a retrospective study, which was inferior to the randomized controlled cohort study in strength. Second, the sample size was relatively small. Third, some parameters (e.g. blood pressure) that might affect oxygenation index were not included in this study.

\section{Conclusions}

In summary, APACHE II score, LIS, SOFA, NUTRIC score, WBC, neutrophils, lymphocyte counts, RAGE, and albumin levels were independent risk factors for severe pneumonia complicated with hypoxemia. RAGE showed a predictive value for severe injury in pneumonia. LIS, SOFA, lactate, lymphocyte, platelet, BUN, total bilirubin, PCT, and OSM levels were independent factors for 30-day mortality. In addition, OSM level upon RICU admission was associated with 30-day mortality.

\section{Abbreviations}

RICU: Respiratory intensive care unit

APACHE II: Acute physiology, age, chronic health evaluation
LIS: $\quad$ Lung injury score; SOFA: sequential organ

failure assessment

NUTRIC The nutrition risk in critically ill score

score:

PCT: Procalcitonin

CRP: $\quad$ C-reactive protein

ARDS: $\quad$ Acute respiratory distress syndrome

CD4: $\quad$ CD4+T cell

CD8: $\quad$ CD8+T cell

CD3: $\quad \mathrm{CD} 3+\mathrm{T}$ cell

$\mathrm{PH}$ : $\quad$ Potential of hydrogen

PaCO2: $\quad$ Partial pressure of arterial carbon dioxide

Lac: $\quad$ Lactic acid

WBC: White blood cell count

BUN: Blood urea nitrogen

LDH: $\quad$ Lactate dehydrogenase

PT: $\quad$ Prothrombin time

APTT: $\quad$ Activated partial thromboplastin time

FiB: $\quad$ Fibrinogen

RAGE: The receptor for advanced glycation end

OSM: $\quad$ Oncostatin $\mathrm{M}$

AUC: $\quad$ Area under the curve

CI: $\quad$ Confidence interval

ROC: Receiver operating characteristic.

\section{Data Availability}

The data used to support the findings of this study are available from the corresponding author upon request.

\section{Disclosure}

This article has been presented as a preprint in Research Square (https://www.researchsquare.com/article/rs-21370/ v1). This study was performed as part of the employment of all co-authors employed by Nanjing First Hospital, Nanjing Medical University.

\section{Conflicts of Interest}

The authors declare that there are no conflicts of interest regarding the publication of this article.

\section{Authors' Contributions}

Jing Lei, Li Wang, and Qian Li have equally contributed to this work.

\section{Supplementary Materials}

The supplementary materials include the following: Word file: STROBE_checklist_v4_combined_PlosMedicine; PDF file: Jiangnan Zhao's consent; PDF file: Yi Shi's consent. (Supplementary Materials)

\section{References}

[1] U. U. Tamhane, S. Aneja, D. Montgomery, E.-K. Rogers, K. A. Eagle, and H. S. Gurm, "Association between admission 
neutrophil to lymphocyte ratio and outcomes in patients with acute coronary syndrome," The American Journal of Cardiology, vol. 102, no. 6, pp. 653-657, 2008.

[2] S. Lattanzi, C. Cagnetti, C. Rinaldi, S. Angelocola, L. Provinciali, and M. Silvestrini, "Neutrophil-to-lymphocyte ratio improves outcome prediction of acute intracerebral hemorrhage," Journal of the Neurological Sciences, vol. 387, pp. 98-102, 2018.

[3] R. Howard, P. A. Kanetsky, and K. M. Egan, "Exploring the prognostic value of the neutrophil-to-lymphocyte ratio in cancer," Scientific Reports, vol. 9, no. 1, Article ID 19673, 2019.

[4] S. Lattanzi, D. Norata, and A. A. Divani, "Systemic inflammatory response index and futile recanalization in patients with ischemic stroke undergoing endovascular treatment," Brain Science, vol. 11, no. 9, 2021.

[5] M. Neeper, A. M. Schmidt, J. Brett et al., "Cloning and expression of a cell surface receptor for advanced glycosylation end products of proteins," Journal of Biological Chemistry, vol. 267, no. 21, pp. 14998-15004, 1992.

[6] M. Jabaudon, E. Futier, L. Roszyk, V. Sapin, B. Pereira, and J.-M. Constantin, "Association between intraoperative ventilator settings and plasma levels of soluble receptor for advanced glycation end-products in patients without pre-existing lung injury," Respirology, vol. 20, no. 7, pp. 1131-1138, 2015.

[7] T. Uchida, M. Shirasawa, L. B. Ware et al., "Receptor for advanced glycation end-products is a marker of type I cell injury in acute lung injury," American Journal of Respiratory and Critical Care Medicine, vol. 173, no. 9, pp. 1008-1015, 2006.

[8] C. S. Calfee, L. B. Ware, M. D. Eisner et al., "Plasma receptor for advanced glycation end products and clinical outcomes in acute lung injury," Thorax, vol. 63, no. 12, pp. 1083-1089, 2008.

[9] B. T. Thompson, R. C. Chambers, and K. D. Liu, "Acute respiratory distress syndrome," New England Journal of Medicine, vol. 377, no. 6, pp. 562-572, 2017.

[10] H. Setiadi, T. Yago, Z. Liu, and R. P. McEver, "Endothelial signaling by neutrophil-released oncostatin $\mathrm{M}$ enhances P-selectin-dependent inflammation and thrombosis," Blood Advances, vol. 3, no. 2, pp. 168-183, 2019.

[11] E. A. Oczypok, T. N. Perkins, and T. D. Oury, "All the "RAGE" in lung disease: the receptor for advanced glycation endproducts (RAGE) is a major mediator of pulmonary inflammatory responses," Paediatric Respiratory Reviews, vol. 23, pp. 40-49, 2017.

[12] A. H. Hergrueter, K. Nguyen, and C. A. Owen, "Matrix metalloproteinases: all the RAGE in the acute respiratory distress syndrome," American Journal of Physiology-Lung Cellular and Molecular Physiology, vol. 300, no. 4, pp. L512L515, 2011.

[13] R. G. Iannitti, A. Casagrande, A. De Luca et al., "Hypoxia promotes danger-mediated inflammation via receptor for advanced glycation end products in cystic fibrosis," American Journal of Respiratory and Critical Care Medicine, vol. 188, no. 11, pp. 1338-1350, 2013.

[14] Z. D. W. Dezman, A. C. Comer, G. S. Smith et al., "Repeat lactate level predicts mortality better than rate of clearance," The American Journal of Emergency Medicine, vol. 36, no. 11, pp. 2005-2009, 2018.

[15] J. F. Bermejo-Martin, C. Cilloniz, R. Mendez et al., "Lymphopenic community acquired pneumonia (L-CAP), an immunological phenotype Associated with higher risk of mortality," EBioMedicine, vol. 24, pp. 231-236, 2017.

[16] O. Arihan, B. Wernly, M. Lichtenauer et al., "Blood Urea Nitrogen (BUN) is independently associated with mortality in critically ill patients admitted to ICU," PLoS One, vol. 13, no. 1, Article ID e0191697, 2018.

[17] R. Zhai, C. C. Sheu, L. Su et al., "Serum bilirubin levels on ICU admission are associated with ARDS development and mortality in sepsis," Thorax, vol. 64, no. 9, pp. 784-790, 2009.

[18] T. Akagi, N. Nagata, H. Miyazaki et al., "Procalcitonin is not an independent predictor of 30-day mortality, albeit predicts pneumonia severity in patients with pneumonia acquired outside the hospital," BMC Geriatrics, vol. 19, no. 1, p. 3, 2019.

[19] N. Peschanski, C. Chenevier-Gobeaux, L. Mzabi et al., "Prognostic value of PCT in septic emergency patients," Annals of Intensive Care, vol. 6, no. 1, p. 47, 2016.

[20] H. Han, Q. Ma, C. Li et al., "Profiling serum cytokines in COVID-19 patients reveals IL- 6 and IL-10 are disease severity predictors," Emerging Microbes \& Infections, vol. 9, no. 1, pp. 1123-1130, 2020.

[21] D. M. Del Valle, S. Kim-Schulze, H.-H. Huang et al., "An inflammatory cytokine signature predicts COVID-19 severity and survival," Nature Medicine, vol. 26, no. 10, pp. 1636-1643, 2020.

[22] K. E. Traber, K. L. Hilliard, E. Allen et al., "Induction of STAT3-dependent CXCL5 expression and neutrophil recruitment by oncostatin-M during pneumonia," American Journal of Respiratory Cell and Molecular Biology, vol. 53, no. 4, pp. 479-488, 2015.

[23] L. Stawski and M. Trojanowska, "Oncostatin M and its role in fibrosis," Connective Tissue Research, vol. 60, no. 1, pp. 40-49, 2019.

[24] P. S. Arunachalam, F. Wimmers, C. K. P. Mok et al., "Systems biological assessment of immunity to mild versus severe COVID-19 infection in humans," Science, vol. 369, no. 6508, pp. 1210-1220, 2020.

[25] P. Li, T. Wang, and M. Chen, "RAGE-mediated functional DNA methylated modification contributes to cigarette smoke-induced airway inflammation in mice," Bioscience Reports, vol. 41, no. 7, 2021. 